\title{
Selection of active plant extracts against the coffee leaf miner Leucoptera coffeella (Lepidoptera: Lyonetiidae)
}

\begin{abstract}
ALVES, D.S. ${ }^{1 *}$; OLIVEIRA, D.F. ${ }^{2}$; CARVALHO, G.A. ${ }^{3}$; CARVALHO, D.A. ${ }^{4}$; SOUZA, L.P. ${ }^{5}$; LASMAR, O. ${ }^{6}$ ${ }^{1}$ Department of Entomology, Federal University of Lavras, C.P. 3037, 37200-000. Lavras/MG. Email: *dejane_bio@ yahoo.com.br; ${ }^{2}$ Department of Chemistry, Federal University of Lavras, C.P. 3037, 37200-000. Lavras/MG. Email: denilson@ufla.br; ${ }^{3}$ Department of Entomology, Federal University of Lavras, C.P. 3037, 37200-000. Lavras/MG. E-mail: gacarval@ufla.br; ${ }^{4}$ Department of Biological Sciences, Federal University of Lavras, C.P. 3037, 37200000. Lavras/MG. E-mail: douglasc@ufla.br; ${ }^{5}$ Department of Chemistry, Federal University of Lavras, C.P. 3037, 37200-000. Lavras/MG. E-mail: Iulousbr@yahoo.com; ${ }^{6}$ Department of Entomology, Federal University of Lavras, Caixa Postal 3037, 37200-000. Lavras/MG. E-mail: olasmar@yahoo.com.br
\end{abstract}

\begin{abstract}
Aiming to contribute to the development of alternative control methods of the coffee leaf miner, Leucoptera coffeella (Guérin-Mèneville \& Perrottet, 1842) (Lepidoptera: Lyonetiidae), a search for plants able to produce active substances against this insect was carried out, with species collected during different periods of time in the Alto Rio Grande region, (Lavras, Minas Gerais, Brazil). Coffee leaves containing $L$. coffeella mines were joined with 106 extracts from 77 plant species and, after 48 hours, the dead and alive caterpillars were counted. The extracts from Achillea millefolium, Citrus limon, Glechoma hederacea, Malva sylvestris, Mangifera indica, Mentha spicata, Mirabilis jalapa, Musa sapientum, Ocimum basiculum, Petiveria alliaceae, Porophyllum ruderale, Psidium guajava, Rosmarinus officinalis, Roupala montana, Sambucus nigra and Tropaeolum majus showed the highest mortality rates.
\end{abstract}

Keywords: Leucoptera coffeella, natural products, botanical insecticide, alternative control

\begin{abstract}
RESUMO: Seleção de extratos de plantas ativos contra o bicho-mineiro-do-cafeeiro Leucoptera coffeella (Lepidoptera: Lyonetiidae). Visando contribuir para o desenvolvimento de métodos alternativos de controle do bicho-mineiro-do-cafeeiro, Leucoptera coffeella (Lepidoptera: Lyonetiidae), buscou-se selecionar plantas coletadas em diferentes épocas na região do Alto Rio Grande, (Lavras, Minas Gerais, Brasil) que contenham substâncias ativas contra este inseto. Folhas de cafeeiro com minas intactas de $L$. coffeella foram colocadas em contato com 106 extratos provenientes de 78 espécies vegetais e, após 48 horas, contaramse as lagartas vivas e mortas. Os extratos de Achillea millefolium, Citrus limon, Glechoma hederacea, Malva sylvestris, Mangifera indica, Mentha spicata, Mirabilis jalapa, Musa sapientum, Ocimum basiculum, Petiveria alliaceae, Porophyllum ruderale, Psidium guajava, Rosmarinus officinalis, Roupala montana, Sambucus nigra e Tropaeolum majus, provocaram os maiores índices de mortalidade.
\end{abstract}

Palavras-chave: Leucoptera coffeella, produtos naturais, inseticida botânico, controle alternativo

\section{INTRODUCTION}

The coffee leaf miner, Leucoptera coffeella (Guérin-Mèneville \& Perrottet, 1842) (Lepidoptera: Lyonetiidae), is considered to be one of the main coffee pests, since the corresponding larvae feed on mesophyllic leaf tissues, resulting in plantation defoliation that may account for an $80 \%$ loss in coffee production (Reis, 1990; Gallo et al., 2002). The most employed methods to control this insect are based on the use of synthetic insecticides, which have not been as efficient as desired to reduce $L$. coffeella population. Furthermore, these products have favored biological imbalances such as the development of secondary pests and have contaminated human beings and the environment with harmful substances (Souza et al., 1998).

A promising alternative to circumvent the previously mentioned problem comprises the use of products from plant sources, since many reports on the activity of plants against insects can be found in the literature (Damarla et al., 2002). An example is

Recebido para publicação em 24/02/2009

Aceito para publicação em 14/11/2012

Rev. Bras. PI. Med., Campinas, v.15, n.3, p.352-362, 2013. 
the effect of the neem seed extract, Azadiracta indica A. Juss on $L$. coffeella, this product has affected the development of larvae and adult emergence of this insect (Venzon et al., 2005). Another example is larvicidal, antifeedant and oviposition deterrent effects of aqueous extract of Argemone mexicana L., Vetiveria zizanioides L. Nash, Annona murricata L., Murraya koenigii (L.) Spreng and Lantana camara L. on the mining insect Plutella xylostella L. (Lepidoptera: Plutellidae) under laboratory and field conditions (Facknath, 2006).

Despite the high potential to produce a great variety of biologically active substances (Taiz \& Zeiger, 2004), most of the plant species found in the Alto Rio Grande region, Minas Gerais State, Brazil have not been submitted to any study aimed to render their biological properties useful for human beings (Rodrigues \& Carvalho, 2001). Consequently, in order to contribute to the development of new methods to control $L$. coffeella, this work aimed at identifying native and exotic plants from that region able to produce substances active against such insect.

\section{MATERIAL AND METHOD}

\section{Plant extracts}

Parts of the plant species (Table 1), collected in the Alto Rio Grande region (Lavras, MG), between October/2001 and May/2005, have been identified by Prof. Douglas A. Carvalho and exsiccata deposited in Herbarium ESAL, at the Biology Department/Federal University of Lavras, Minas Gerais State, Brazil. Considering the lack of studies with plants from the Alto do Rio Grande region the work aimed to utilize species with known insecticidal activity and also with which studies are lacking, in order to provide greater insight into the biological potential of the region. They were cut into small pieces with scissors and submitted to extraction with methanol at room temperature, for 48 hours. The resulting mixtures were filtered through cotton and residues were extracted with methanol once more. The liquids from both extractions were combined, concentrated to dryness in a rotatory evaporator and freeze-dried. For 13 plant species collected between May/2004 and June/2004 (Table 1 ), no freeze-drying was carried out to avoid a loss of volatiles (Bos et al., 2002).

\section{Test with the coffee leaf miner}

To perform the experiment with $L$. coffeella, an aloquot $(0.070 \mathrm{~g})$ of each freeze-dried plant extract was dissolved in $8.0 \mathrm{~mL}$ of an aqueous $1.0 \%(\mathrm{~g} \mathrm{~mL}$ 1) Tween 80 solution. Regarding plant extracts not submitted to the freeze-drying process, the volume $(\mathrm{mL})$ of Tween 80 solution was ten times the mass (g) of fresh plant used. Coffee plant leaves (Coffea arabica $L$. cv. Topázio) from a $L$. coffeella containing greenhouse, with intact mines of the insect, were dipped (10 seconds) into the extracts solution and maintained in a chamber at $25 \pm 1^{\circ} \mathrm{C}$, with $70 \% \mathrm{RH}$, and $14 \mathrm{~h}$ photoperiod, for 48 hours. Then, mines were opened to count dead and live larvae. Aqueous 1.0\% $\left(\mathrm{g} \mathrm{mL}^{-1}\right.$ ) Tween 80 and $0.2 \%\left(\mathrm{~g} \mathrm{~mL}^{-1}\right)$ Sumithion ${ }^{\circledR} 500$ CE solutions were employed as negative and positive controls, respectively. The experiments were carried out in a randomized design, with four replicates per treatment, each composed of five mines. Values were transformed into percentages before statistical procedures, which comprised analyses of variances (ANOVA) and comparison of means in accordance with the Scott \& Knott (1974) test at 5\%. The software SISVAR was used to do so (SISVAR, 2000).

TABLE 1. Plant species collected in the Alto Rio Grande region (Lavras-MG) and used for the extract preparation.

\begin{tabular}{|c|c|c|c|c|c|}
\hline \multirow[t]{2}{*}{ Family/Scientific name } & \multicolumn{2}{|c|}{ Common name } & \multirow{2}{*}{$\begin{array}{l}\text { Date of collection } \\
\qquad(\mathrm{dd} / \mathrm{mm} / \mathrm{yy})\end{array}$} & \multirow{2}{*}{$\begin{array}{c}\text { Part } \\
\text { collected }\end{array}$} & \multirow{2}{*}{$\begin{array}{l}\text { Voucher } \\
\text { number }\end{array}$} \\
\hline & Portuguese & English & & & \\
\hline \multicolumn{6}{|l|}{ Anacardiaceae } \\
\hline Schinus molle L. & Arueira & Pepper tree & $25 / 06 / 02$ & Leaves & 14800 \\
\hline Mangifera indica L. & Manga & Mango & 03/01/05 & Leaves & 1945 \\
\hline \multicolumn{6}{|l|}{ Annonaceae } \\
\hline Annona cacans Warm. & Araticum-cagão & - & $12 / 03 / 02$ & Leaves & 12787 \\
\hline \multicolumn{6}{|l|}{ Apiaceae } \\
\hline Foeniculum vulgare Mill. & Funcho & Fennel & 18/10/01 & Leaves & 5319 \\
\hline \multicolumn{6}{|l|}{ Asteraceae } \\
\hline Achillea millefolium L. & Mil-folhas & Milfoil & 02/10/01 & nflorescences & 17618 \\
\hline Achillea millefolium L. & Mil-folhas & Milfoil & 02/10/01 & Leaves & 17618 \\
\hline Achillea millefolium L. & Mil-folhas & Milfoil & 09/11/04 & Leaves & 17618 \\
\hline
\end{tabular}

Rev. Bras. PI. Med., Campinas, v.15, n.3, p.352-362, 2013. 
TABLE 1. Plant species collected in the Alto Rio Grande region (Lavras-MG) and used for the extract preparation.

\begin{tabular}{|c|c|c|c|c|c|}
\hline \multirow[t]{2}{*}{ Family/Scientific name } & \multicolumn{2}{|c|}{ Common name } & \multirow{2}{*}{$\begin{array}{l}\text { Date of collection } \\
\qquad(\mathrm{dd} / \mathrm{mm} / \mathrm{yy})\end{array}$} & \multirow{2}{*}{$\begin{array}{c}\text { Part } \\
\text { collected }\end{array}$} & \multirow{2}{*}{$\begin{array}{l}\text { Voucher } \\
\text { number }\end{array}$} \\
\hline & Portuguese & English & & & \\
\hline Arctium lappa L. & Barbana & Burdock & $10 / 07 / 02$ & Leaves & 19826 \\
\hline Artemisia vulgaris $\mathrm{L}$. & Artemísia & Mugwort & $16 / 08 / 02$ & Leaves & 19817 \\
\hline Baccharis dracunculifolia D.C. & Alecrim-do-campo & Baccharis & 09/08/02 & Leaves & 15866 \\
\hline Calea hispida Baker & Erva-de-lagarto & - & $12 / 03 / 02$ & Leaves & 11517 \\
\hline Calendula officinalis $\mathrm{L}$. & Calendula & - & $01 / 11 / 04$ & Leaves & 9449 \\
\hline Chenopodium ambrosiodes L. & Erva-de-santa-maria & Epazote & $12 / 09 / 02$ & Leaves & 9747 \\
\hline${ }^{1}$ Chenopodium ambrosioides $\mathrm{L}$. & Erva-de-santa-maria & Epazote & $20 / 05 / 04$ & Leaves & 9747 \\
\hline Cynara scolymus L. & Cinarina & Artichoke & $18 / 10 / 01$ & Leaves & 9736 \\
\hline Gochnatia barrosil Cabrera & - & - & $26 / 10 / 01$ & Leaves & 12075 \\
\hline Porophyllum ruderale (Jacq.) Cass. & Arnica -paulista & Poreleaf & 19/09/02 & Leaves & 13441 \\
\hline${ }^{1}$ Porophyllum ruderale (Jacq.) Cass. & Arnica-paulista & Poreleaf & $20 / 05 / 04$ & Leaves & 13441 \\
\hline Tagetes sp. & - & - & $22 / 02 / 05$ & Leaves & 16070 \\
\hline Taraxacum officinale Wiggers & Dente-de-leão & Dandelion & $22 / 10 / 02$ & Leaves & 7316 \\
\hline Tithonia diversifolia (Hemsl) Gray & Girasol & Mexican sunflower & $26 / 09 / 02$ & Leaves & 10027 \\
\hline \multicolumn{6}{|l|}{ Burseraceae } \\
\hline Protium heptaphyllum (Aubl.) March & Protium & Protium & $26 / 10 / 01$ & Leaves & 13602 \\
\hline \multicolumn{6}{|l|}{ Caprifoliaceae } \\
\hline Sambucus nigra L. & Sabugueiro & Black lace & 05/07/02 & Leaves & 4605 \\
\hline${ }^{1}$ Sambucus nigra L. & Sabugueiro & Black lace & $20 / 05 / 04$ & Leaves & 4605 \\
\hline \multicolumn{6}{|l|}{ Clethraceae } \\
\hline Clethra scabra Pers. & Caujuja & Clethra & $26 / 10 / 01$ & Leaves & 17453 \\
\hline \multicolumn{6}{|l|}{ Clusiaceae } \\
\hline Kielmeyera coriacea Mart. ex Saddi. & Pau-Santo & - & $12 / 03 / 02$ & Leaves & 7975 \\
\hline \multicolumn{6}{|l|}{ Curcubitaceae } \\
\hline Curcuma longa L. & Açafrão & Tumeric & $16 / 12 / 02$ & Leaves & 19814 \\
\hline Momordica charantia L. & Melão-de-são-caetano & Bitter gourd & $12 / 09 / 02$ & Leaves & 8856 \\
\hline Momordica charantia L. & Melão-de-são-caetano & Bitter gourd & $22 / 05 / 04$ & Leaves & 8856 \\
\hline \multicolumn{6}{|l|}{ Dilleniaceae } \\
\hline Davilla elliptica St. Hill & Lixinha & Cofferaria & $23 / 11 / 01$ & Leaves & 9725 \\
\hline \multicolumn{6}{|l|}{ Ebenaceae } \\
\hline Diospyros hispida D. C. & Bacupari bravo & - & $12 / 03 / 02$ & Leaves & 8930 \\
\hline \multicolumn{6}{|l|}{ Equisetaceae } \\
\hline Equisetum arvense L. & Cavalinha & - & $16 / 02 / 05$ & Stems & 2028 \\
\hline \multicolumn{6}{|l|}{ Erythroxylaceae } \\
\hline Erythroxylum suberosum St.-Hill. & Mercúrio-do-campo & - & $26 / 10 / 01$ & Leaves & 8631 \\
\hline \multicolumn{6}{|l|}{ Euphorbiaceae } \\
\hline Croton antisyphiliticus Mart. & Canela-de-perdiz & - & $26 / 10 / 01$ & Leaves & 13382 \\
\hline \multicolumn{6}{|l|}{ Euphorbiaceae } \\
\hline Phyllanthus tenellus Roxb. & Quebra-pedra & Long-stalk & $28 / 06 / 02$ & Leaves & 12620 \\
\hline \multicolumn{6}{|l|}{ Fabaceae } \\
\hline Andira anthelmia (Vell.) JF Macbr. & Angelim-de- morcego & Cabbage tree & $12 / 03 / 02$ & Leaves & 7790 \\
\hline Mimosa pudica L. & Dormideira & - & $16 / 02 / 05$ & Flowers & 6190 \\
\hline Senna obtusifolia (L.) Irwin \& Barneby & Fedegoso & Java bean & $16 / 12 / 02$ & Leaves & 8072 \\
\hline
\end{tabular}

Rev. Bras. PI. Med., Campinas, v.15, n.3, p.352-362, 2013. 
TABLE 1. Plant species collected in the Alto Rio Grande region (Lavras-MG) and used for the extract preparation.

\begin{tabular}{|c|c|c|c|c|c|}
\hline \multirow[t]{2}{*}{ Family/Scientific name } & \multicolumn{2}{|c|}{ Common name } & \multirow{2}{*}{$\begin{array}{l}\text { Date of collection } \\
\qquad(\mathrm{dd} / \mathrm{mm} / \mathrm{yy})\end{array}$} & \multirow{2}{*}{$\begin{array}{c}\text { Part } \\
\text { collected }\end{array}$} & \multirow{2}{*}{$\begin{array}{l}\text { Vouche } \\
\text { number }\end{array}$} \\
\hline & Portuguese & English & & & \\
\hline \multicolumn{6}{|l|}{ Senna rugosa (G. Don.) } \\
\hline H. S. Irwin \& Barneby & Senna & - & 03/04/02 & Flowers & 13914 \\
\hline \multicolumn{6}{|l|}{ Senna rugosa (G. Don.) } \\
\hline H. S. Irwin \& Barneby & Senna & - & 03/04/02 & Leaves & 13914 \\
\hline \multicolumn{6}{|l|}{ Lamiaceae } \\
\hline Glechoma hederacea L. & Hera-terrestre & Ground ivy & $11 / 10 / 01$ & Leaves & 6886 \\
\hline${ }^{1}$ Glechoma hederacea L. & Hera-terrestre & Ground ivy & $20 / 05 / 04$ & Leaves & 6886 \\
\hline Leonorus sibiricus $\mathrm{L}$. & Erva macaé & Mother wort & $16 / 08 / 02$ & Leaves & 4345 \\
\hline Mentha spicata L. & Hortelã-peluda & Spearmint & $10 / 07 / 02$ & Leaves & 11666 \\
\hline Mentha spicata L. & Hortelã-peluda & Spearmint & 02/10/01 & Leaves & 11666 \\
\hline${ }^{1}$ Mentha spicata L. & Hortelã-peluda & Spearmint & $28 / 06 / 04$ & Leaves & 11666 \\
\hline Mentha spicata L. & Hortelã-peluda & Spearmint & $16 / 02 / 05$ & Leaves & 11666 \\
\hline Ocimum basiculum L. & Basilicão & - & $16 / 02 / 05$ & Leaves & 17890 \\
\hline Ocimum gratissimum $\mathrm{L}$. & Alfavaca & - & $16 / 02 / 05$ & Leaves & 10807 \\
\hline Peltodon tomentosus Pohl. & Hortelã-do-mato & - & 03/04/02 & Inflorescences & 6168 \\
\hline Peltodon tomentosus Pohl. & Hortelã-do-mato & - & 03/04/02 & Leaves & 6168 \\
\hline Rosmarinus officinalis $\mathrm{L}$. & Alecrim & Rosemary & 02/10/01 & Leaves & 3130 \\
\hline Tetradenia riparia (Hochst.) Codd & Mirra & Misty plume Bush & $22 / 10 / 02$ & Leaves/Stems & 19811 \\
\hline Thymus vulgaris $\mathrm{L}$. & Tomilho & - & $22 / 02 / 05$ & Leaves & 17057 \\
\hline \multicolumn{6}{|l|}{ Liliaceae } \\
\hline Allium cepa L. & Cebola & Onion & $19 / 09 / 02$ & Leaves & 930 \\
\hline Allium sativum $\mathrm{L}$. & Alho & Garlic & $22 / 10 / 02$ & Leaves & 19824 \\
\hline Aloe vera $\mathrm{L}$. & Babosa & True aloe & 02/08/02 Leav & aves without slin & e19820 \\
\hline${ }^{1}$ Aloe vera $\mathrm{L}$. & Babosa & True aloe & 08/06/04 Leav & aves without slin & e19820 \\
\hline \multicolumn{6}{|l|}{ Lythraceae } \\
\hline Diplusodon virgatus Pohl. & Cai-cai & - & 19/10/01 & Leaves & 10601 \\
\hline \multicolumn{6}{|l|}{ Malpighiaceae } \\
\hline \multicolumn{6}{|l|}{ Banisteriopsis campestris (A. Juss.) } \\
\hline Little. & - & - & $12 / 03 / 02$ & Leaves & 14436 \\
\hline \multicolumn{6}{|l|}{ Malvaceae } \\
\hline Malva sylvestris L. & Malva & Common mallow & $25 / 06 / 02$ & Leaves & 19815 \\
\hline Malva sylvestris L. & Malva & Common mallow & 02/10/01 & Branches & 19815 \\
\hline${ }^{1}$ Malva sylvestris $\mathrm{L}$. & Malva & Common mallow & $28 / 06 / 04$ & Leaves & 19815 \\
\hline Peltaea polymorpha St. Hil. Krap. & - & - & $23 / 11 / 01$ & Leaves & 13940 \\
\hline \multicolumn{6}{|l|}{ Melastomataceae } \\
\hline Marcetia taxifolia (A. St.-Hil.) DC. & Marcetia & - & $26 / 10 / 01$ & Leaves & 14416 \\
\hline Miconia albicans (Sw.) Triana. & Quaresmeira-branca & - & $26 / 10 / 01$ & Leaves & 18045 \\
\hline \multicolumn{6}{|l|}{ Meliaceae } \\
\hline Cabralea canjerana (Vellozo) Martius. & Canjerana & - & $26 / 10 / 01$ & Leaves & 18048 \\
\hline \multicolumn{6}{|l|}{ Moraceae } \\
\hline Ficus carica L. & Figueira & - & $19 / 11 / 04$ & Leaves & 2146 \\
\hline
\end{tabular}


TABLE 1. Plant species collected in the Alto Rio Grande region (Lavras-MG) and used for the extract preparation.

\begin{tabular}{|c|c|c|c|c|c|}
\hline \multirow[t]{2}{*}{ Family/Scientific name } & \multicolumn{2}{|c|}{ Common name } & \multirow{2}{*}{$\begin{array}{l}\text { Date of collection } \\
\text { (dd/mm/yy) }\end{array}$} & \multirow{2}{*}{$\begin{array}{c}\text { Part } \\
\text { collected }\end{array}$} & \multirow{2}{*}{$\begin{array}{l}\text { Voucher } \\
\text { number }\end{array}$} \\
\hline & Portuguese & English & & & \\
\hline \multicolumn{6}{|l|}{ Musaceae } \\
\hline Musa sapientum & Bananeira & Banana & $18 / 07 / 02$ & Leaves & 14727 \\
\hline Musa sapientum & Bananeira & Banana & $14 / 05 / 05$ & Leaves & 14727 \\
\hline \multicolumn{6}{|l|}{ Myrtaceae } \\
\hline \multicolumn{6}{|l|}{ Campomanesia pubescens (DC.) } \\
\hline O. Berg & Guabiroba & - & $26 / 10 / 01$ & Leaves & 14043 \\
\hline Myrcia fallax (Rich.) DC & Guamarim-do-preto & - & $12 / 03 / 02$ & Leaves & 17857 \\
\hline Psidium guajava L. & Goiabeira & Guava & 03/01/05 & Leaves & 2129 \\
\hline \multicolumn{6}{|l|}{ Nictaginaceae } \\
\hline Mirabilis jalapa L. & Maravilha & Clavillia & $18 / 10 / 01$ & Leaves & 8333 \\
\hline${ }^{1}$ Mirabilis jalapa L. & Maravilha & Clavillia & $20 / 05 / 04$ & Leaves & 8333 \\
\hline \multicolumn{6}{|l|}{ Ochnaceae } \\
\hline Ouratea spectabilis (Mart.) Engl. & Folha-de-serra & - & $26 / 10 / 01$ & Leaves & 8632 \\
\hline \multicolumn{6}{|l|}{ Phytolaccaceae } \\
\hline Petiveria alliaceae L. & Guiné & Anamu & 04/10/02 & Leaves & 5127 \\
\hline${ }^{1}$ Petiveria alliacea $\mathrm{L}$. & Guiné & Anamu & $28 / 06 / 04$ & Leaves & 5127 \\
\hline Petiveria sp. & - & - & $16 / 12 / 02$ & Leaves & 10302 \\
\hline \multicolumn{6}{|l|}{ Plantaginacea } \\
\hline Plantago tomentosa L. & Tanchagem & - & 02/08/02 & Leaves & 7756 \\
\hline \multicolumn{6}{|l|}{ Poaceae } \\
\hline Coix-lacrima jobi L. & Capim-rosário & Job's tears & 19/10/01 & Leaves & 5994 \\
\hline Coix- lacrima jobi L. & Capim-rosário & Job's tears & $22 / 02 / 05$ & Leaves & 5994 \\
\hline Echinolaena inflexa (Poir.) Chase & Capim-flexa & - & $12 / 03 / 02$ & Branches & 14449 \\
\hline${ }^{1}$ Echinolaena inflexa (Poir.) Chase & Capim-flexa & - & $04 / 06 / 04$ & Branches & 14449 \\
\hline \multicolumn{6}{|l|}{ Polygalaceae } \\
\hline Polygala angulata DC. Brasil & - & - & $12 / 03 / 02$ & Leaves & 13603 \\
\hline \multicolumn{6}{|l|}{ Proteaceae } \\
\hline Roupala montana Aubl. & Carne-de-vaca & Grilled meat & $26 / 10 / 01$ & Leaves & 8786 \\
\hline${ }^{1}$ Roupala montana Aubl. & Carne-de-vaca & Grilled meat & 04/06/04 & Leaves & 8786 \\
\hline \multicolumn{6}{|l|}{ Punicaceae } \\
\hline Punica granatum $\mathrm{L}$. & Romã & Pomegranate & $11 / 10 / 01$ & Leaves & 2182 \\
\hline${ }^{1}$ Punica granatum $\mathrm{L}$. & Romã & - & $28 / 06 / 04$ & Leaves & 2182 \\
\hline Punica granatum $\mathrm{L}$. & Romã & - & 03/01/05 & Leaves & 2182 \\
\hline \multicolumn{6}{|l|}{ Rubiaceae } \\
\hline Alibertia sessilis (Vell.) K. Schum. & Marmelinho & - & $26 / 10 / 01$ & Leaves & 15275 \\
\hline Rudgea virbunoides Benth & Chá-de-bugre & - & $26 / 10 / 01$ & Leaves & 14001 \\
\hline \multicolumn{6}{|l|}{ Rutaceae } \\
\hline Citrus aurantium L. & Laranja-amarga & - & $28 / 03 / 05$ & Leaves & 2248 \\
\hline Citrus limon (L.) Burm. F. & Limão & - & 03/01/05 & Leaves & 19825 \\
\hline Citrus sp. & - & - & $18 / 07 / 02$ & Leaves & 20763 \\
\hline Ruta graveolens L. & Arruda & Rue & 02/10/01 & Leaves & 3133 \\
\hline Salicaceae & & - & & & \\
\hline Casearia sylvestris Sw. & Guaçatonga & Wild coffee & $26 / 10 / 01$ & Leaves & 17461 \\
\hline
\end{tabular}


TABLE 1. Plant species collected in the Alto Rio Grande region (Lavras-MG) and used for the extract preparation.

...continuação

\begin{tabular}{|c|c|c|c|c|c|}
\hline \multirow[t]{2}{*}{ Family/Scientific name } & \multicolumn{2}{|c|}{ Common name } & \multirow{2}{*}{$\begin{array}{l}\text { Date of collection } \\
\qquad(\mathrm{dd} / \mathrm{mm} / \mathrm{yy})\end{array}$} & \multirow{2}{*}{$\begin{array}{l}\text { Part } \\
\text { collected }\end{array}$} & \multirow{2}{*}{$\begin{array}{l}\text { Voucher } \\
\text { number }\end{array}$} \\
\hline & Portuguese & English & & & \\
\hline \multicolumn{6}{|l|}{ Sapindaceae } \\
\hline Serjania erecta Raldlk & Timbó & - & $26 / 10 / 01$ & Leaves & 7591 \\
\hline${ }^{1}$ Serjania erecta Raldlk & Timbó & - & $28 / 05 / 04$ & Leaves & 7591 \\
\hline \multicolumn{6}{|l|}{ Solanaceae } \\
\hline Nicotiana tabacum L. & Tabaco & Tobacco & 07/11/02 & Leaves & 6343 \\
\hline \multicolumn{6}{|l|}{ Tropaeolaceae } \\
\hline Tropaeolum majus L. & Capuchinha & Garden nasturtium & $18 / 10 / 01$ & Leaves & 8853 \\
\hline Tropaeolum majus L. & Capuchinha & Garden nasturtium & 18/10/01 & Flowers & 8853 \\
\hline Tropaeolum majus L. & Capuchinha & Garden nasturtium & 02/08/02 & Leaves & 8853 \\
\hline${ }^{1}$ Tropaeolum majus L. & Capuchinha & Garden nasturtium & 20/05/04 & Leaves & 8853 \\
\hline Tropaeolum majus L. & Capuchinha & Garden nasturtium & $14 / 02 / 05$ & Flowers & 8853 \\
\hline \multicolumn{6}{|l|}{ Zingiberaceae } \\
\hline Zingiber officinale Roscoe & Gengibre & - & $19 / 11 / 04$ & Leaves & 2297 \\
\hline
\end{tabular}

\section{RESULT AND DISCUSSION}

Among the freeze-dried extracts from plants collected between October/2001 and December/2002, those from Achillea millefolium, Allium cepa, Allium sativum, Aloe vera, Arctium lappa, Artemisia vulgaris, Banisteriopsis campestris, Chenopodium ambrosioides, Citrus sp., Croton antisiphyliticus, Curcuma longa, Foeniculum vulgare, Mentha spicata, Momordica charantia, Nicotiana tabacum, Petiveria sp., Punica granatum, Ruta graveolens, Schinus molle and Tithonia diversifolia, did not affect the $L$. coffella survival, though these plants were reported as active against other insects (Alexander et al., 1991; Dutta et al., 1993; Franzios et al., 1997; Adedire \& Lajide, 1999; Larocque et al., 1999; Botha \& Mccrindle, 2000; Hadis et al. 2003; Fuchs \& Bowers, 2004; Khattak et al., 2004; Moshi et al., 2004; Jaenson et al., 2005; Pavela, 2005; Traboulsi et al., 2005; Yildirim et al., 2005; Ferrero, 2006; Gökce et al., 2006; Han et al., 2006; Kathuria \& Kaushik, 2006; Mao et al., 2006; Mitchell \& Ahmad, 2006; Zong \& Wang, 2007).

Perhaps the results were different because the plants show variation in the production of secondary metabolites, according to the climatic conditions in which they were grown (Gobbo-Neto \& Lopes, 2007). Moreover, different species of insects have different mechanisms for detoxification against the same substance, caffeine, for example, blocks the development of Aedes aegypti (Diptera: Culicidae), but does not have an adverse effect on Perileucoptera :coffeella (Lepidoptera: Lyonetiidae) (Guerreiro Filho \& Mazzafera, 2000; Laranja et al., 2006). One must also consider the conditions of extraction, given the fact that secondary plant metabolites include several classes of substances. For example, for the extraction of polar compounds it is common to use methanol, ethanol or water an extractor. For nonpolar substances, hexane is a good extractor. In this sense, the results found in this study can be partly attributed to the different extraction conditions. Ferrero et al. (2006), for example, working with the hexane extract of S. molle, characterized by solubilizing nonpolar substances, while in the present study methanol extract was worked with. The mode of application of plant extract is also another factor that influences the results, Pascual-Villalobos \& Fernández (1999) found that extracts of squill bulbs (Urginea maritima (L.) Baker) topically applied caused greater mortality than when compared with those that were mixed in the diet.

The freeze-dried extracts of the following plants were also inactive against $L$. coffeella: Alibertia sessilis, Andira anthelmia, Annona cacans, Baccharis dracunculifolia, Cabralea canjerana, Calea hispida, Campomanesia pubescens, Casearia sylvestris, Clethra scabra, Coix-lacrima jobi, Cynara scolymus, Davilla elliptica, Diospyros hispida, Diplusodon virgatus, Echinolaena inflexa, Erythroxylum suberosum, Gochnatia barrosil, Kielmeyera coriacea, Leonorus sibiricus, Marcetia taxifolia, Miconia albicans, , Myrcia fallax, Ouratea spectabilis, Peltaea polymorpha, Peltodon tomentosus, Phyllanthus tenellus, Plantago tomentosa, Polygala angulata, Protium heptaphyllum, Rudgea virbunoides, Senna obtusifolia, Senna rugosa, Serjania erecta, 
Taraxacum officinale and Tetradenia riparia. Such a result seemed reasonable, since no report about the insecticidal properties of these plants could be found in the literature.

Differently from the previously mentioned results, the freeze-dried extracts of Glechoma hederacea, Malva sylvestris, Mirabilis jalapa, Petiveria alliaceae, Porophyllum ruderale, Rosmarinus officinalis, Sambucus nigra and Tropaeolum majus, increased L. coffeella larvae mortality (Table 2), corroborate the activity of such plants against other insects (Cammue et al., 1992; Huang \& Renwick, 1995; Manea, 1995; Guillet el al., 1998; Chariandy et al., 1999; Guarrera, 2005; Jaenson et al., 2005; Miresmailli \& Isman, 2006; Singh et al., 2006). Some of these plants have insecticidal activity against other insects of the order Lepidoptera. The essential oil of $R$. officinalis affects the survival of Pseudaletia unipuncta Haworth (Noctuidae) and Trichoplusia ni Hübner (Noctuidae), the terpenoid group of compounds were attributed to the deleterious activity of this plant against these insects (Isman et al., 2008). Another example is the feeding deterrent activity of T. majus on Pieris rapae, attributed mainly due to the presence of chlorogenic acid (Huang \& Renwick, 1995). Regarding Roupala montana, the extract of which presented activity against $L$. coffeella, no report was found in the literature about the insecticidal activity of such plant. There are few studies with $R$. montana, but in plants belonging to the family Proteaceae, the presence of substances active against insects of the order Lepidoptera, such as, bisresorcinol derivatives, polyphenolic compounds and coumarins is reported in the literature (Hadaek et al, 1994; Erazo et al., 1997; Verotta et al., 1999; Koppera et al., 2002; Wang et al., 2009).

Despite of the increase in L. coffeella larvae mortality by freeze-dried extracts from $G$. hederacea, M. jalapa, M. sylvestris., T. majus (flowers), P. alliaceae, R. montana, P. ruderale and $S$. nigra colleted during the years of 2001 and 2002, extracts from the same plants collected between November/2004 and May/2005, prepared without employing the freeze-drying process, were all inactive. Since the freeze-dried extracts should be less active due to loss of volatiles, metabolic variations caused by environmental changes (Beppu et. al, 2004; Kofidis et al., 2004) probably accounted for the absence of insecticidal properties.

Among those plants whose freeze-dried extracts from parts collected during the years of 2001 and 2002 were inactive, A. millefolium e M. spicata were randomly selected for a new collection and preparation of freeze-dried extracts, which increased L. coffeella larvae mortality (Table 3 ). Conversely, S. nigra and T. majus (Table 3), lost their activity
TABLE 2. Effect of freeze-dried extracts from plants collected in the Alto Rio Grande region (Lavras-MG), between October/2001 and December/2002, on Leucoptera coffeela larvae mortality.

\begin{tabular}{|c|c|c|}
\hline Plant species & $\begin{array}{c}\text { Part } \\
\text { collected }\end{array}$ & $\begin{array}{c}\text { Larvae } \\
\text { mortality (\%) }\end{array}$ \\
\hline \multicolumn{3}{|l|}{ Experiment 1} \\
\hline Foeniculum vulgare & leaves & $24.8 \mathrm{a}$ \\
\hline Glechoma hederacea & leaves & $44.8 \mathrm{~b}$ \\
\hline Mirabilis jalapa & leaves & $49.2 \mathrm{~b}$ \\
\hline Ruta graveolens & leaves & $9.5 \mathrm{a}$ \\
\hline Tropaeolum majus & flowers & $44.4 \mathrm{~b}$ \\
\hline Sumithion 500 CE (control) & & $100.0 \mathrm{c}$ \\
\hline Tween 80 (control) & & $5.56 \mathrm{a}$ \\
\hline \multicolumn{3}{|l|}{ Experiment 2} \\
\hline Malva sylvestris & branches & $62.7 \mathrm{~b}$ \\
\hline Punica granatum & leaves & $34.4 \mathrm{a}$ \\
\hline Tween 80 (control) & & $14.3 \mathrm{a}$ \\
\hline Sumithion $500 \mathrm{CE}$ (control) & & $100.0 \mathrm{c}$ \\
\hline \multicolumn{3}{|l|}{ Experiment 3} \\
\hline Marcetia taxifolia & leaves & $10.5 \mathrm{a}$ \\
\hline Peltaea polymorpha & leaves & $8.8 \mathrm{a}$ \\
\hline Rosmarinus officinalis & leaves & $27.9 \mathrm{~b}$ \\
\hline Tween 80 (control) & & $7.7 \mathrm{a}$ \\
\hline Sumithion $500 \mathrm{CE}$ (control) & & $100.0 \mathrm{c}$ \\
\hline \multicolumn{3}{|l|}{ Experiment 4} \\
\hline Arctium lappa & leaves & $16.2 \mathrm{a}$ \\
\hline Baccharis dracunculifolia & leaves & $14.6 \mathrm{a}$ \\
\hline Echinolaena inflexa & branches & $34.0 \mathrm{a}$ \\
\hline Gochnatia barrosil & leaves & $14.2 \mathrm{a}$ \\
\hline Petiveria alliaceae & leaves & $58.7 \mathrm{~b}$ \\
\hline Tween 80 (control) & & $14.6 \mathrm{a}$ \\
\hline Sumithion 500 CE (control) & & $100.0 \mathrm{c}$ \\
\hline \multicolumn{3}{|l|}{ Experiment 5} \\
\hline Leonorus sibiricus & leaves & $17.7 \mathrm{a}$ \\
\hline Phyllanthus tenellus & leaves & $8.1 \mathrm{a}$ \\
\hline Roupala montana & leaves & $30.2 \mathrm{~b}$ \\
\hline Tween 80 (control) & & $13.3 \mathrm{a}$ \\
\hline Sumithion $500 \mathrm{CE}$ (control) & & $100.0 \mathrm{c}$ \\
\hline \multicolumn{3}{|l|}{ Experiment 6} \\
\hline Davilla elliptica & leaves & $7.1 \mathrm{a}$ \\
\hline Kielmeyera coriacea & leaves & $6.7 \mathrm{a}$ \\
\hline Porophyllum ruderale & leaves & $42.4 \mathrm{~b}$ \\
\hline Sambucus nigra & leaves & $35.8 \mathrm{~b}$ \\
\hline Tropaeolum majus & leaves & $17.7 \mathrm{a}$ \\
\hline Tween 80 (control) & & $8.6 \mathrm{a}$ \\
\hline Sumithion 500 CE (control) & & $100.0 \mathrm{c}$ \\
\hline
\end{tabular}

(1) Means followed by the same letter in each experiment do not differ significantly $(P \leq 0.05)$. 
when a new plant collection and extract preparation was carried out. Once more, variations in metabolic production by plants due to environmental changes may account for these results (Gobbo-Neto \& Lopes, 2007; Vila-Verde et al., 2005). P. lanceotata can be used to exemplify such behavior. According to Bowers et al. (1992), only during the summer can aucubin and catalpol, which are the insecticides produced by that plant, be detected in the leaves.

Among the freeze-dried extracts from plants collected between October/2004 and May/2005, the one from Citrus limon was active (Table 3 ). This result corroborates the activity against $A$ tta sexdens rubropilosa Forel, 1908 (Hymenoptera: Formicidae)

TABLE 3. Effect of freeze-dried extracts from plants collected in the Alto Rio Grande Region (LavrasMG), between November/2004 and May/2005, on Leucoptera coffeella larvae mortality.

\begin{tabular}{|c|c|c|}
\hline Plant species & $\begin{array}{c}\text { Part } \\
\text { collected }\end{array}$ & $\begin{array}{c}\text { Larvae } \\
\text { mortality (\%) }\end{array}$ \\
\hline \multicolumn{3}{|l|}{ Experiment 1} \\
\hline Achillea millefolium & flowers & $26.3 \mathrm{~b}$ \\
\hline Aloe vera & leaves & $8.5 \mathrm{a}$ \\
\hline Citrus limon & leaves & $38.6 \mathrm{~b}$ \\
\hline Glechoma hederacea & leaves & $14.1 \mathrm{a}$ \\
\hline Mangifera indica & leaves & $27.4 \mathrm{~b}$ \\
\hline Mentha spicata & leaves & $31.0 \mathrm{~b}$ \\
\hline Musa sapientum & leaves & $37.7 \mathrm{~b}$ \\
\hline Ocimum basiculum & leaves & $43.2 \mathrm{~b}$ \\
\hline Psidium guajava & leaves & $23.5 b$ \\
\hline Zingiber officinale & leaves & $12.3 \mathrm{a}$ \\
\hline Tween 80 (control) & & $8.5 \mathrm{a}$ \\
\hline Sumithion $500 \mathrm{CE}$ (control) & & 99.7 c \\
\hline \multicolumn{3}{|l|}{ Experiment 2} \\
\hline Equisetum arvense & leaves & $0.0 \mathrm{a}$ \\
\hline Malva sylvestris & leaves & $0.0 \mathrm{a}$ \\
\hline Mirabilis jalapa & leaves & $7.1 \mathrm{a}$ \\
\hline Ocimum gratissimum & leaves & $0.0 \mathrm{a}$ \\
\hline Petiveria alliaceae & leaves & $13.7 \mathrm{a}$ \\
\hline Roupala montana & leaves & $5.8 \mathrm{a}$ \\
\hline Sambucus nigra & leaves & $0.0 \mathrm{a}$ \\
\hline Tagetes sp. & leaves & $0.0 \mathrm{a}$ \\
\hline Thymus vulgaris & leaves & $2.8 \mathrm{a}$ \\
\hline Tropaeolum majus & leaves & $0.0 \mathrm{a}$ \\
\hline Tween 80 (control) & & $0.0 \mathrm{a}$ \\
\hline Sumithion 500 CE (control) & & $100.0 \mathrm{c}$ \\
\hline
\end{tabular}

reported by Fernandes et al. (2002). Analogously, the freeze-dried extracts from Mangifera indica, Musa sapientum, Ocimum basiculum and Psidium guajava also increased $L$. coffeella larvae mortality (Table $3)$, though no report on their insecticidal properties could be found.

Despite of the reports on the ability to produce insecticidal substances by Calendula officinalis, Mimosa pudica, Ocimum gratissimum, Punica granatum, Tagetes sp., Thymus vulgaris and Zingiber officinale (Regnaultroger \& Hamraoui, 1993; Williams \& Mansingh, 1993; Huang \& Renwick, 1995; Larocque et al. 1999; Navickiene et al., 2003; Aslan et al., 2004; Sarin, 2004; Seri-Kouassi et al., 2004; Aslan et al., 2005; Guarrera, 2005, Prajapati el at., 2005; Traboulsi et al., 2005), no effect on L. coffeella was observed for the freeze-dried extract from parts of these plants collected between October/2004 and May/2005.

Regarding the freeze-dried extracts of Citrus aurantium, Coix-lacrima jobi, Equisetum arvense and Ficus carica, also collected between October/2004 and May/2005, no influence on L. coffeella could be observed, which is in accordance with the absence of reports in the literature on the insecticidal activity of such plants.

As the presence of chemical groups in plants that have proven active against $L$. coffeella in this work, amides isolated from $A$. millefolium with high insecticidal activity against Aedes triseriatus (Say) larvae (LaLonde et al., 1980) stand out. C. limon is reported to have a high proportion of limonene and in lower quantities p-menthane molecules and pinenes in its composition, that are active against insects such as Culex pipiens (Diptera: Culicidae) (Michaelakis et al., 2009). G. hederacea has lecithin in its leaves that has insecticidal activity against Leptinotarsa decemlineata (Wang et al., 2003). $M$. sylvestris produces, among other secondary metabolites, terpenoids and derivatives and phenol, which in turn, are classes of substances known to have insecticidal activity (Cutillo et al., 2005; Geris et al., 2008). Although no reports were found for the insecticidal activity of $M$. indica, the presence of compounds with known insecticidal activity, such as saponins, steroids, tannis, flavonoids, reducing sugars, cardiac glycosides and anthraquinone have already been detected (Aiyelaagbe \& Osamudiamen, 2009). Among the main compounds found in $M$. spicata, carvone deserves mention, which also has insecticidal activity (Tripathi et al., 2003; Chauhana et al., 2009). While trypsin inhibitors have been isolated from $M$. jalapa , such compounds may cause negative effects on the survival of insects (Kowalska et al., 2007; Kansal et al., 2008). In the case of $P$. alliaceae, the essential oil obtained from the roots of this plant proved to be active against Bemicia

Rev. Bras. PI. Med., Campinas, v.15, n.3, p.352-362, 2013. 
tabaci. The major constituent isolated from the oil, benzaldehyde, was also active against this insect (Bezerra, 2006). The mono and sesquiterpenes and fatty acid derivatives of $P$. ruderale active against Ostrinia nubilalis (Lepidoptera: Pyralidae may also be cited. In this context, insecticidal activity of $R$. officinalis on Pseudaletia unipuncta. Haworth (Noctuidae) and the Trichoplusia ni. Hübner (Noctuidae) was attributed to the terpenoid constituents. Regarding S. nigra, the activity against Hemipteran insect species was attributed to protein agglutinin I (SNA-I). It is a Type-2 ribosomeinactivating proteins (RIPs) (Shahidi-Noghabi et al, 2009). It is also possible to mention that the leaves of $T$. majus contain high amounts of the glucosinolate glucotropaeolin, this substance is known to have an insecticidal effect (Kleinwchter et al., 2008; Peterson et al., 1998).

Concluding, in this work the activity against $L$. coffeella larvae was detected for the freezedried extracts from $A$. millefolium, C. limon, $G$. hederacea, $M$. sylvestris, $M$. indica, $M$. spicata, $M$. jalapa, M. sapientum, O. basiculum, $P$. alliaceae, $P$. ruderale, $P$. guajava, $R$. officinalis, $R$. montana, $S$. nigra and T. majus. Nevertheless, the production of substances active against the insect by such plants during the whole year can not be guaranteed, since environmental conditions may affect metabolic production by plant species.

\section{ACKNOWLEDGEMENT}

The authors are grateful to Conselho Nacional de Desenvolvimento Científico e Tecnológico (CNPq), Coordenação de Aperfeiçoamento de Pessoal de Nível Superior (CAPES), Fundação de Amparo à Pesquisa do Estado de Minas Gerais (FAPEMIG) and to Programa Nacional de Pesquisa e Desenvolvimento do Café (PNP \& D-Café), for financial support and fellowships.

\section{REFERENCE}

ADEDIRE, C.O.; LAJIDE, L. Toxicity and oviposition deterrency of some plant extracts on cowpea storage bruchid, Callosobruchus maculatus Fabricius. Journal of Plant Diseases and Protection, v.106, n.6, p.64753, 1999.

AIYELAAGBE, O.O.; OSAMUDIAMEN, P.M. Phytochemical screening for active compounds in Mangifera indica leaves from Ibadan, Oyo State. Plant Sciences Research, v.2, n.1, p.11-13, 2009.

ALEXANDER, I.C. et al. An insecticidal diterpene from Croton-linearis. Phytochemistry, v.30, n.6, p.1801-3, 1991.

ASLAN, I. et al. Toxicity of essential oil vapours to two greenhouse pests, Tetranychus urticae Koch and Bemisia tabaci Genn. Industrial Crops and Products, v.19, n.2, p.167-73, 2004.
ASLAN, I. et al. Toxicity of the vapours of Artemisia absinthium essential oils to Tetranychus urticae Koch and Bemisia tabaci (Genn.). Fresenius Environmental Bulletin, v.14, n.5, p.413-17, 2005.

BEPPU, $H$. et al. Studies on the components of Aloe arborescens from Japan - monthly variation and differences due to part and position of the leaf. Biochemical Systematics and Ecology, v.32, n. 9, p.783-95, 2004.

BEZERRA, J.N.S. Composição química, atividade fitonematicida e inseticidade Tipi (Petiveria alliaceae). 2006. 90p. Dissertação (Mestrado - Área de Concentração Química Orgânica). Departamento de Química Universidade Federal do Ceará, Fortaleza.

BOS, R. et al. Volatile components from Anthriscus sylvestris (L.) Hoffm. Journal of Chromatografy, v.966, n.1-2, p.233-38, 2002.

BOTHA, B.M.; MCCRINDLE, C.M.E. An appropriate method for extracting the insect repellent citronellol from an indigenous plant (Pelargonium graveolens L'Her) for potential use by resource-limited animal owners. Journal of the South African Veterinary Association - Tydskrif Van Die Suid-Afrikaanse Veterinere Vereniging, v.71, n.2, p.103-5, 2000.

BOWERS, M. D. et al. Effects of genotype, habitat, and seasonal-variation on iridoid glycoside content of Plantago lanceolata (Plantaginaceae) and the implications for insect herbivores. Oecologia, v.91, n.2, p.201-7, 1992.

CAMMUE, B.P.A. et al. Isolation and characterization of a novel class of plant antimicrobial peptides from Mirabilis jalapa L seeds. Journal of Biological Chemistry, v.267, n.4, p.2228-33, 1992.

CHARIANDY, C.M. et al. Screening of medicinal plants from Trinidad and Tobago for antimicrobial and insecticidal properties. Journal of Ethnopharmacology, v.64, n.3, p.265-70, 1999.

CHAUHANA, R.S. et al. Chemical composition of essential oils in Mentha spicata L. accession [IIIM(J)26] from North-West Himalayan region, India. Industrial Crops and Products, v.29, n.2-3, p.654-656, 2009.

CUTILLO, F. et al. Terpenoids and phenol derivatives from Malva silvestris. Phytochemistry, v.67, n.5, p.481-485, 2005.

DAMARLA, S.R. et al. Patente $\mathbf{n}^{\circ} \mathbf{6 . 3 4 0 . 4 8 4}$. United States Patent. Jan / 2002.

DUTTA, P. et al. Persistent efficacy of the feeding deterrents of Tithonia diversifolia (Hemsl) Gray against Diacrisia obliqua Walker (Lepidoptera, Arctiidae). Journal of Environmental Biology, v.14, n.2, p.10712, 1993.

ERAZO, S. et al. Phytochemical and biological study of radal Lomatia hirsuta (Proteaceae). Journal of Ethnopharmacology, v.57, n.2, p.81-83, 1997.

FERNANDES, J.B. et al. Extrações de óleos de sementes de citros e suas atividades sobre a formiga cortadeira Atta sexdens e seu fungo simbionte. Química nova, v.25, n.6, p.1091-5, 2002.

FERRERO A. A. et al. Biological activity of Schinus molle on Triatoma infestans. Fitoterapia, v.7, n.5, p.381-83, 2006.

FRANZIOS, G. et al. Insecticidal and genotoxic activities of mint essential oils. Journal of Agricultural and Food 
Chemistry, v.45, n.7, p.2690-4, 1997.

FUCHS, A.; BOWERS, M.D. Patterns of iridoid glycoside production and induction in Plantago lanceolata and the importance of plant age. Journal of Chemical Ecology, v.30, n.9, p.1723-41, 2004.

GALLO, D. et al. Manual de entomologia agrícola. 2.ed. São Paulo: Agronômica Ceres, 2002. 649p.

GERIS, R. et al. Larvicidal effects of fungal meroterpenoids in the control of Aedes aegypti L., the main vector of dengue and yellow fever. Chemistry and Biodiversity, v.5, n.2, p. 341-345, 2008.

GOBBO-NETO, L.; LOPES, N. P. Plantas Medicinais: Fatores de Influência no Conteúdo de Metabólitos Secundários. Química Nova, v.30, n.2, p.374-81, 2007.

GÖKCE, A., et al. Behavioural response of Colorado potato beetle (Leptinotarsa decemlineata) larvae to selected plant extracts. Pest Management Science, v.62, n.11, p.1052-1057, 2006.

GUARRERA, P. M. Traditional phytotherapy in Central Italy (Marche, Abruzzo, and Latium). Fitoterapia, v.76, n.1, p.1-25, 2005.

GUERREIRO FILHO O.; MAZZAFERA P. Caffeine does not protect coffee against the leaf miner Perileucoptera coffeella. Journal of Chemical Ecology, v.26, n.3, p.1447-1464, 2000.

GUILLET, G. et al. Volatile monoterpenes in Porophyllum gracile and $P$. ruderale (Asteraceae): Identification, localization and insecticidal synergism with alphaterthienyl. Phytochemistry, v.49, n.2, p.423-29, 1998.

HADAEK, F. et al. Analysis, isolation and insecticidal activity of linear furanocoumarins and other coumarin derivatives from Peucedanum (Apiaceae: Apioideae). Journal of Chemical Ecology, v.20, n.8, 1994.

HADIS, M. et al. Field trials on the repellent activity of four plant products against mainly Mansonia population in western Ethiopia. Phytotherapy Research, v.17, n.3, p.202-5, 2003.

HAN, M.K. et al. Insecticidal and antifeedant activities of medicinal plant extracts against Attagenus unicolor japonicus (Coleoptera : Dermestidae). Journal of Stored Products Research, v.42, n.1, p.15-22, 2006.

HUANG, X.P.; RENWICK, J. A. A. Cross habituation to feeding deterrents and acceptance of a marginal host-plant by Pieris-rapae larvae. Entomologia Experimentalis et Applicata, v.76, n.3, p. 295-302, 1995.

ISMAN, M.B. et al. Insecticidal activities of commercial rosemary oils (Rosmarinus officinalis) against larvae of Pseudaletia unipuncta and Trichoplusia ni in relation to their chemical compositions. Pharmaceutical Biology, v.46, n.1-2, p.82-87, 2008.

FACKNATH, S. Effects of phytoextracts and natural enemy to control Plutella xylostella L. (Lepidoptera: Plutellidae) in cabbage. Allelopathy Journal, v.17, n.2, p.207-222, 2006.

JAENSON T.G.T. et al. Evaluation of extracts and oils of tick-repellent plants from Sweden. Medical and Veterinary Entomology, v.19, n.4, p.345-52, 2005.

KANSAL, R. et al. Purification and characterization of trypsin inhibitor from Cicer arietinum L. and its efficacy against Helicoverpa armigera. Brazilian Journal of Plant Physiology, v.20, n.4, 2008.

KATHURIA, V.; KAUSHIK, N. Evaluation of insecticidal property of some plant species against Helicoverpa armigera. Indian Journal of Agricultural Sciences, v.76, n.10, p.614-17, 2006.

KHATTAK, S. et al. In vitro screening for biological and pharmacological effects of indigenous medicinal plants, Mentha Iongifolia and Aloe vera. Journal of the Chemical Society of Pakistan, v.26, n.3, p.24851, 2004.

KLEINWCHTER, M. et al. The glucosinolate"myrosinase system in nasturtium (Tropaeolum majus L.): variability of biochemical parameters and screening for clones feasible for pharmaceutical utilization. Journal of Agricultural and Food Chemistry, v.56, n.23, p.1116511170, 2008.

KOFIDIS, G. et al. Seasonal variation of essencial oils in a linalool-rich chemotype of Mentha spicata grown wild in Greece. Journal of Essential Oil Research, v.16, n.5, p.469-72, 2004.

KOPPERA, B.J. et al. Effects of paper birch condensed tannin on whitemarked tussock moth (Lepidoptera: Lymantriidae) performance. Environmental Entomology, v.31, n.1, p.10-14, 2002.

KOWALSKA, J. et al. Trypsin inhibitors from the garden four o'clock (Mirabilis jalapa) and spinach (Spinacia oleracea) seeds: Isolation, characterization and chemical synthesis , Phytochemistry, v.68, n.11, p.1487-1496, 2007.

LALONDE, R. T. et al. N-(2-methylpropyl)-(E,E)-2,4decadienamide a mosquito larvicide from Achillea millefolium L. Journal of Chemical Ecology, v.6, n.1, p.35-48, 1980.

LARANJA, A.T. et al. Caffeine effect on mortality and oviposition in successive generations of Aedes aegypti. Revista de Saude Pública, v.40, v.6, p.1112-1117, 2006.

LAROCQUE, N. et al. Effects of tansy essential oil from Tanacetum vulgare on biology of oblique-banded leafroller, Choristoneura rosaceana. Journal of Chemical Ecology, n.25, v.6, p.1319-30, 1999.

MANEA, L. Plants, active substances and natural medicines in Calendula officinalis. Revista de Chimie, v.46, n.12, p.9-10, 1995.

MAO, L.X. et al. Vetiver oil and nootkatone effects on the growth of pea and citrus. Industrial Crops and Products, v.23, n.3., p.327-32, 2006.

MICHAELAKIS, A. et al. Citrus essential oils and four enantiomeric pinenes against Culex pipiens (Diptera: Culicidae). Parasitology Research, v.105, n.3, p.769773, 2009.

MIRESMAILLI, S.; ISMAN, M.B. Efficacy and persistence of rosemary oil as an acaricide against twospotted spider mite (Acari: Tetranychidae) on greenhouse tomato. Journal of Economic Entomology, v.99, n.6, p.2015-23, 2006.

MITCHELL, S.A.; AHMAD, M.H. A review of medicinal plant research at the University of the West Indies, Jamaica, 1948-2001. West Indian Medical Journal, v.55, n.4, p.243-69, 2006.

MOSHI, M.J. et al. Testing beyond ethnomedical claims: Brine shrimp lethality of some Tanzanian plants. Pharmaceutical Biology, v.42, n.7, p.547-51, 2004.

NAVICKIENE, H.M.D. et al. Quantitative determination of anti-fungal and insecticide amides in adult

Rev. Bras. PI. Med., Campinas, v.15, n.3, p.352-362, 2013. 
plants, plantlets and callus from Piper tuberculatum by reverse-phase high-performance liquid chromatography. Phytochemical Analysis, v.14, n.5, p.281-84, 2003.

PASCUAL-VILLALOBOS, M.J.; FERNÁNDEZ, M. Insecticidal activity of ethanolic extracts of Urginea maritima (L.) Baker bulbs. Industrial Crops and Products, v.10, n.2, p. 115-120, 1999.

PAVELA, R. Insecticidal activity of some essential oils against larvae of Spodoptera littoralis. Fitoterapia, v.76, n.7-8, p.691-96, 2005.

PETERSON, C.J. et al. Glucosinolate aglucones and analogues: insecticidal properties and a QSAR. Pesticide Science, v.54,n.1, p.35-42,1998.

PRAJAPATI, V. et al. Insecticidal, repellent and ovipositiondeterrent activity of selected essential oils against Anopheles stephensi, Aedes aegypti and Culex quinquefasciatus. Bioresource Technology, v.96, n.16, p.1749-57, 2005.

REGNAULTROGER, C.; HAMRAOUI, A. Efficiency of plants from the south of France used as traditional protectants of Phaseolus vulgaris $L$ against its bruchid Acanthoscelides obtectus (Say). Journal of Stored Products Research, v.29, n.3, p.259-64, 1993.

REIS, P.R. Manejo integrado das pragas do cafeeiro em Minas Gerais. In: FERNANDES, O. A. et al. (eds). Manejo integrado de pragas e nematóides. Jaboticabal: FUNEP, 1990. p.39-57.

RODRIGUES, V. E. G.; CARVALHO, D. A. Plantas medicinais no domínio do cerrado. 1.ed. Lavras: UFLA, 2001, 180 p.

SARIN, R. Insecticidal activity of callus culture of Tagetes erecta. Fitoterapia, v.75, n.1, p.62-4, 2004.

SCOTT, A.J.; KNOTT, M.A. A cluster analyses method for grouping means in the analyses of variance. Biometrics, v.30, p.502-12, 1974.

SERI-KOUASSI, B.P. et al. Action of essential oils from two aromatic plants from vory Coast on Callosobruchus maculatus F. of cowpea. Comptes Rendus Chimie, v.7, n.10-11, p.1043-6, 2004.

SINGH, T. et al. Carbohydrate specificity of an insecticidal lectin isolated from the leaves of Glechoma hederacea towards mammalian glycoconjugates. Febs Journal, v.273, p.263-63, 2006.

SISVAR, Sistema para análise de variância, para Windows versão 4.3. Lavras, Universidade Federal de Lavras, 2000.
SHAHIDI-NOGHABI, S. et al. Expression of Sambucus nigra agglutinin (SNA-I') from elderberry bark in transgenic tobacco plants results in enhanced resistance to different insect species. Transgenic Research, v.18, n.2, p.249-592, 2009.

SOUZA, J.C. et al. Bicho-mineiro do cafeeiro: biologia, danos e manejo integrado. 2.ed. Belo Horizonte: EPAMIG, 1998. 48p.

TAIZ, L.; ZEIGER, E. Fisiologia vegetal. 3.ed. Porto Alegre: Artmed, 2004. 719p.

TRABOULSI, A.F. et al. Repellency and toxicity of aromatic plant extracts against the mosquito Culex pipiens molestus (Diptera: Culicidae). Pest Management Science, v.61, n.6, p.597-604, 2005.

TRIPATHI, A.K. et al. Bioactivities of l-carvone, d-carvone, and dihydrocarvone toward three stored product beetles. Journal of Economic Entomology, v.96, n.5, p.1594-1601, 2003.

VENZON, M. et al. The potential of NeemAzal for the control of coffee leaf pests. Crop Protection, v.24, n.3, p.213-219, 2005.

VEROTTA, L. et al. Polyphenolic glycosides from african proteaceae. Journal of Natural Products, v.62, n.11, p.1526-1531, 1999.

VILA-VERDE, G.M. et al. Estudo farmacognóstico das folhas do Anis-do-cerrado, Croton Aff. Zehntneri Pax and H. Hoffm coletado em Serranópolis-GO. Revista Eletrônica de Farmácia, v.2, p.232-35, 2005.

WANG, W. et al. The Tn antigen-specific lectin from ground ivy is an insecticidal protein with an unusual physiology. Plant Physiology , v.132, n.3, p.13221334, 2003.

WANG, H. Prenylated bisresorcinols from Grevillea floribunda. Phytochemistry Letters v.2, n.1, p.41-45, 2009.

WILLIAMS, L.A.D.; MANSINGH, A. Pesticidal potentials of tropical plants insecticidal activity in leaf extracts of 60 plants. Insect Science and its Application, v.14, n.5-6, p.697-700, 1993.

YILDIRIM, E. et al. The effects of essential oils from eight plant species on two pests of stored product insects. Fresenius Environmental Bulletin, v.14, n.1, p.23-7, 2005.

ZONG, N.; WANG, C.Z. Larval feeding induced defensive responses in tobacco: comparison of two sibling species of Helicoverpa with different diet breadths. Planta, v.226, n.1, p.215-24, 2007. 